\title{
SPECIFIC OF INTERACTION OF THE MACROPARTICLES WITH THE PLASMA-BEAM SYSTEMS
}

\author{
A.A. Bizyukov', A.D. Chibisov ${ }^{2}$, E.V. Romashchenko ${ }^{1}$, V.V. Masich ${ }^{2}$ \\ ${ }^{1}$ V.N. Karazin Kharkiv National University, Kharkiv, Ukraine; \\ ${ }^{2}$ H.S. Skovoroda Kharkiv National Pedagogical University, Kharkiv, Ukraine
}

The presented paper summarizes the results of the last works of the authors on modeling the processes of heating and evaporation of macroparticles in a plasma-beam system. The emphasis are made on the influence of the parameters of the plasma as well as the electron beam introduced into the plasma on these processes. A detailed analysis of the effects that accelerate or slow down the rate of heating and evaporation of macroparticles has been performed.

PACS: $52.40 . \mathrm{Hf}$

\section{INTRODUCTION}

Dusty plasma is an ionized gas that contains micrometer sized particles that is called dust particles or macroparticles (MP). For the first time, dusty plasma was obtained by Langmuir in the $20 \mathrm{~s}$ of the 20th century. As a result of the active development of plasma technologies, such as controlled thermonuclear fusion, plasma etching, ion-plasma deposition of coatings, dusty plasma has become one of the most studying fields of plasma physics. In technological processes such as plasma etching, ion-plasma deposition of coatings, MP are a negative factor and lead to contamination of surfaces, the formation of roughness, and weakening of adhesion of coatings to the surface. Such particles can be formed during the operation of plasma sources, as a result of the interaction of plasma with surfaces inside the chamber. Regarding this, the development of methods for removing of MP from plasma is an actual problem. This paper presents a review of works where the analysis of the conditions under which the evaporation of MP is possible as a result of their interaction with plasma has been performed [1-4].

\section{HEATING AND EVAPORATION OF MP IN PLASMA-BEAM SYSTEMS}

In [5], the effect of the parameters of a gas-discharge plasma, as well as the parameters of an electron beam, on the equilibrium temperature of a single MP and on its potential, has been studied. The heating of the MP and the process of its charging are related processes due to the significant thermionic emission current at high temperatures of the MP; for this purpose, a set of equations (balance of energies and balance of currents) has been solved. The set of the equations takes into account the effect of these two processes on each other:

$$
\left\{\begin{array}{l}
I_{i}^{p l}+I_{e}^{p l}+I_{e}^{b}+I_{e}^{s}+I_{e}^{t h}=0 \\
P_{e}^{p l}+P_{i}^{p l}+P_{e}^{b}-P_{\mathrm{s}}-P_{r}-P_{t h}=0 .
\end{array}\right.
$$

The processes of charging and heating of the MPs associated with the absorption of plasma particles is described by the OML theory and have the form:

$$
\begin{gathered}
I_{i}^{p l}\left(\varphi_{a}\right)=e Z<n_{0} v_{i(e)} \sigma_{i(e)}^{O M L}>=e Z \cdot \Gamma_{i}, \\
I_{e}^{p l}\left(\varphi_{a}\right)=e<n_{0} v_{i(e)} \sigma_{i(e)}^{O M L}>=e \cdot \Gamma_{e},
\end{gathered}
$$

where $\sigma_{i(e)}^{O M L}$ is the cross section of absorption of the ions (electrons), $e$ is the elementary charge, $n_{0}$ is the plasma density,

$$
\begin{gathered}
\Gamma_{i}=\sqrt{8 \pi} a^{2} n_{0} v_{T i}\left(1-\frac{Z e \varphi_{a}}{k T_{i}}\right), \\
\Gamma_{e}=\sqrt{8 \pi} a^{2} n_{0} v_{T e} \exp \left(-\frac{e \varphi_{a}}{k T_{e}}\right),
\end{gathered}
$$

$v_{T i(e)}$ is the ion (electron) thermal velocity $v_{T e}=\sqrt{k T_{e} / m_{e}}, \quad v_{T i}=\sqrt{k T_{i} / m_{i}}, \quad \varphi_{a}$ is a floating MP potential. Electron beam current is given by:

$$
I_{e}^{b}=e \cdot \Gamma_{e}^{b}=\pi a^{2} n_{b} e \sqrt{\frac{k \varepsilon_{b}}{m_{e}}} \cdot\left(1+\frac{2 e \varphi_{a}}{m_{e} v_{e}^{2}}\right),
$$

where $\varepsilon_{b}$ is the energy of the electron beam. Secondary electron emission is given by the formula:

$$
I_{e}^{s}=\delta I_{e}^{b},
$$

where $\delta=\delta_{\max } \frac{k \varepsilon_{b}-e \varphi_{a}}{k E_{m}} \exp \left(2\left(1-\sqrt{\frac{k \varepsilon_{b}-e \varphi_{a}}{k E_{m}}}\right)\right)$ is the secondary emission yield: $\varepsilon_{b}$ is the kinetic energy of primary electron, $E_{m}$ is the electron energy that gives the maximum of secondary emission yield $\delta_{\max }$.

Thermionic emission current is given by the Richardson formula.

$$
I_{e}^{t h}=4 \pi a^{2} A T_{a}^{2} \exp \left(\frac{e \Phi-\Delta W}{k_{B} T_{a}}\right),
$$

where $A=\frac{4 \pi m_{e} k_{B}^{2} e}{h^{3}}, h$ is the Planck constant, $k_{B}$ is the Stefan-Boltzmann constant, $e \Phi$ is the work function, $T_{a}$ is the temperature of the MP. $\Delta W=\sqrt{e^{3} \varphi_{a} / a}$ is the decreasing of the electron work function (Schottky effect). 
The second equation of (1) describes of changing of MP temperature caused by energy flows the following processes: $P_{i(e)}^{p l}$ is the energy flow of plasma particles to the MP; $P_{e}^{b}$ is the energy flow of electron beam; $P_{r}$ is the energy radiated from the MP surface; $P_{e v p r}$ is the cooling due to evaporation of MP substance; $P_{t h}$ is the energy flow from the MP surface is transferred by the electrons of thermionic current; $P_{e}^{s}$ is the energy flow due to the secondary electron emission. The values of the respective energy flows are determined by the following relations:

$$
\begin{gathered}
P_{e}^{p l}=\Gamma_{e} \cdot\left(2 k T_{e}+e \Phi\right), \\
P_{i}^{p l}=\Gamma_{i} \cdot\left(2 k T_{i}+e \varphi+Z I+e \Phi\right), \\
P_{e}^{b}=\Gamma_{e}^{b} \cdot\left(k \varepsilon_{e}+e \Phi\right), \\
P_{r}=\sigma T^{4}, \\
P_{t h}=\Gamma_{e}^{t h} \cdot\left(2 k_{b} T_{a}\right), \\
P_{e}^{s}=\Gamma_{s} \cdot\left(<\varepsilon_{s}>+e \Phi\right),
\end{gathered}
$$

$P_{a}^{\Sigma}=P_{e}^{p l}+P_{i}^{p l}+P_{e}^{b}-P_{\mathrm{s}}-P_{r}-P_{t h}$ is the total energy on the MP, it can be positive or negative. $\Gamma_{e}^{\text {th }}=I_{e}^{\text {th }} / e$, $\Gamma_{s}=I_{s}^{e-e} / e,\left\langle\varepsilon_{s}\right\rangle$ is the averaged energy of the secondary electrons, $I$ is the energy of the ionization of ion. During the solving of the set of equations (1), the plasma density, as well as the energy of the electron beam have been being varied as a result, the dependences of the equilibrium MP temperature that can be reached and the related potential have been obtained. The computation results are shown in Figs. 1, 2.

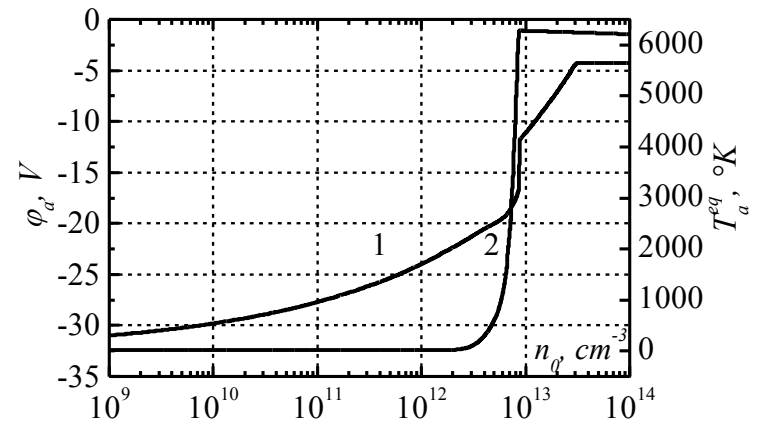

Fig. 1. Equilibrium temperature (1) and related floating potential (2) of the tungsten MP

An analysis of the solution of the set of equations (1) shows that the process of thermionic emission has a significant effect on the value of the floating potential at temperatures greater than $2000 \mathrm{~K}$, that is reached at plasma densities $n_{0}$ greater than $2 \cdot 10^{12} \mathrm{~cm}^{-3}$, while the absolute magnitude of the MP potential is decreased to the values near to zero. In a case when the electron beam injected into the plasma, a change of the magnitude of the MP potential is occurred at lower plasma densities $10^{9} \ldots 10^{1} \mathrm{l} \mathrm{cm}^{-3}$, the energy of the electron beam is $\varepsilon_{\mathrm{e}} \mathrm{b} \approx(0.5 \ldots 0.7) \mathrm{keV}$ this effect is due to an additional mechanism of energy exchange. Simulation has shown that the increasing of the plasma density, as well as the energy of the electron beam, leads to intense heating of the MP.
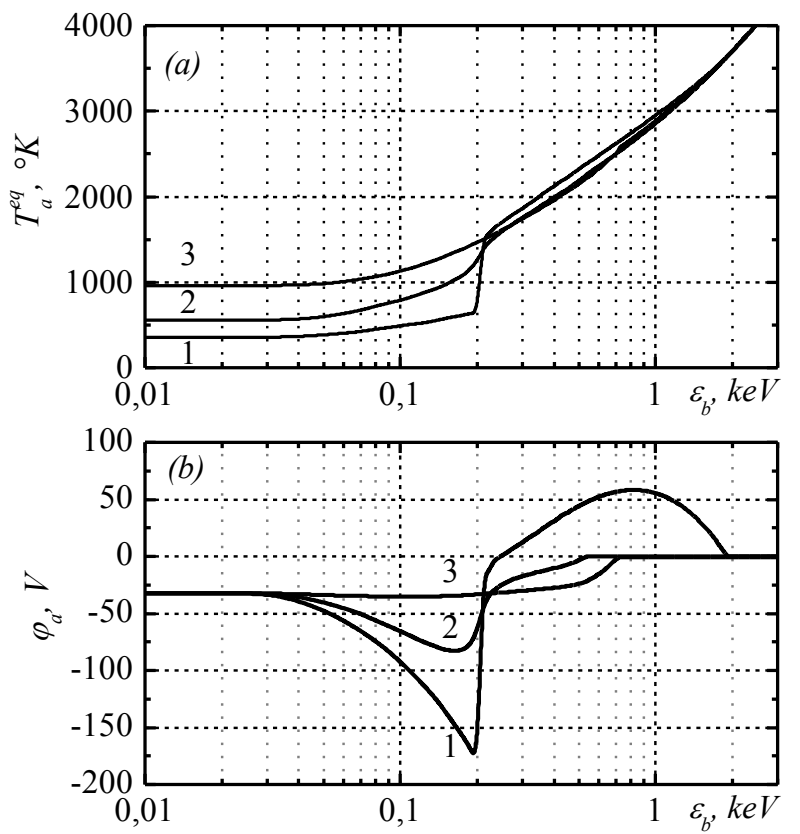

Fig. 2. Equilibrium temperature (1) and related floating potential (2) of the tungsten MP as a function of the energy of electron beam:

$1-n_{0}=10^{9} \mathrm{~cm}^{-3} ; 2-n_{0}=10^{10} \mathrm{~cm}^{-3}$; $3-n_{0}=10^{11} \mathrm{~cm}^{-3}$

In [6], the interaction of the MP with the lowpressure arc discharge plasma is considered. The key feature of such plasma is multiply charged ions, as well as directional component of velocity of the ion species. The dependence of the floating potential and the temperature of the MP on the plasma parameters are determined by the set of equations (1). The fluxes of plasma ions and electrons to the MP surface are described by the OLM theory similarly to the [5] and given by the expressions (2)-(12). The solution of the set of equations (1) in the case of multiply charged ions for a molybdenum MP is presented in Fig. 3. An analysis of the solution of the set of equations shows that in the plasma of a low-pressure arc discharge, the floating MP potential and equilibrium temperature of the MP is significantly depend on the energy that is released on the surface of the MP during ion recombination.

The evaporation time of MPs of radius $a$ in the case of multiply charged ions is described by relation:

$$
t_{b}=4 \pi \rho \int_{0}^{a} \frac{r^{2} d r}{H P_{a}^{\Sigma}\left(T_{a}^{b}\right)} .
$$

$H$ is a specific heat of vaporization, $\rho$ is a MP substance density. 
The numerical computation of the evaporation time of the molybdenum MP (Fig. 4) show that in plasma of density $10^{14} \mathrm{~cm}^{-3}$, for molybdenum some of the MPs can be vaporized, for instance it has been shown that vaporization times of the MPs with typical sizes of 1 and $10 \mu \mathrm{m}$, are $10^{-3}$ and $2 \cdot 10^{-2} s$ respectively.
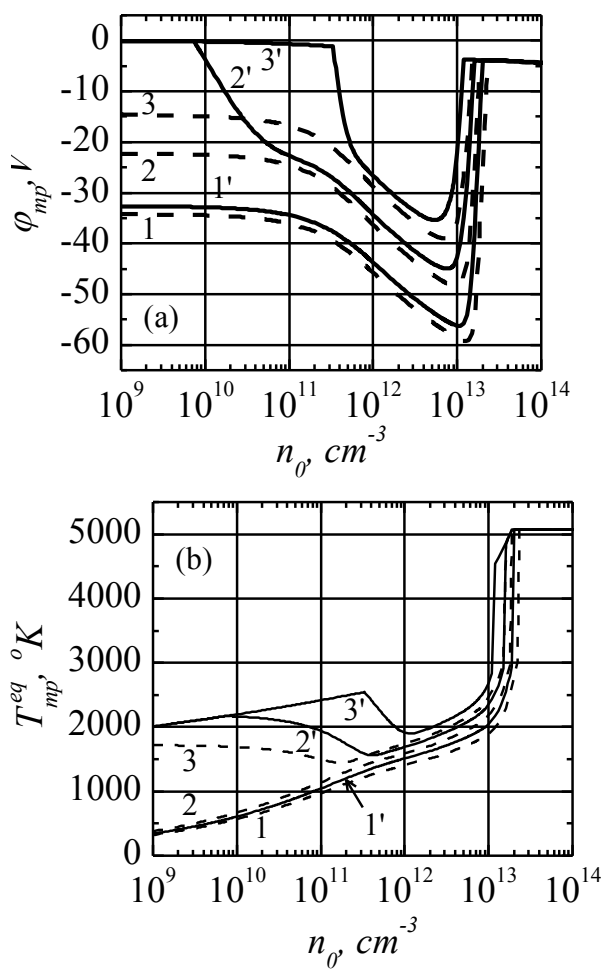

Fig. 3. Floating potential (a) and related equilibrium temperature (b) of the molybdenum MP as a function of plasma density:

$1,1^{\prime}-Z=1 ; 2,2^{\prime}-Z=2 ; 3,3^{\prime}-Z=3$; dashed lines

$1,2,3-20 \mathrm{eV}$, solid lines $1,2,3-35 \mathrm{eV}$

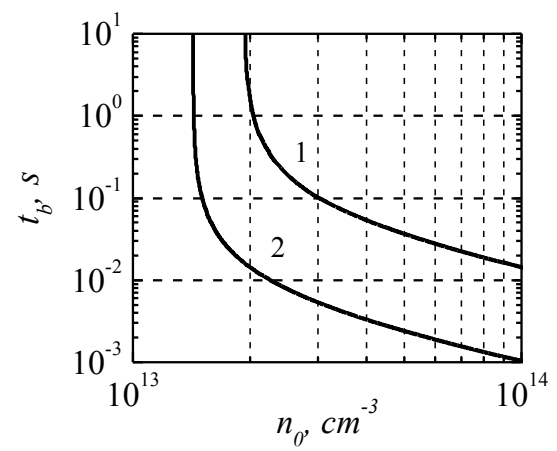

Fig. 4. Evaporation time of the molybdenum MP:

$1-1 \mu \mathrm{m}, 2-10 \mu \mathrm{m}$ averaged over the ion energy

Computations also show the fact that if the plasma density is increased then the vaporization time is decreased. Thus, it has been shown that increasing of plasma density lead to creating conditions when the evaporation of the MPs is possible. It has been shown that multiply charged ions lead to more intensive heating of the MP.
In [7], the process of decay of liquid MPs due to Rayleigh instability that can occurs as a result of charging of the MPs up to high absolute magnitude of the electric charge by the high-energy electron beam in plasma has been studied. To study the possibility of MP decay, the time needed to occurring of Rayleigh instability and the time when the MP is charged have been compared. To solve this problem, unlike the papers $[5,6]$ where the energy balance equation has been solved, in this paper the dynamic problem of finding the charge and the temperature of the MP has been solved. The set of equations that describes these processes has the form

$$
\left\{\begin{array}{l}
I_{e}^{p l}\left(\varphi_{a}\right)+I_{e}^{b}-I_{e}^{s}\left(\varphi_{a}\right)-I_{e}^{t h}\left(\varphi_{a}, T_{a}\right)-I_{i}^{p l}\left(\varphi_{a}\right)=a \frac{d \varphi_{a}}{d t}, \\
P_{e}^{p l}+P_{e}^{b}+P_{i}^{p l}-P_{e}^{s}-P_{r}-P_{e v p r}-P_{t h}=m_{M P} c \frac{d T}{d t} .
\end{array}\right.
$$

The terms that included in the set of equations have the forms (2)-(13), the term describes the loose of energy as a result of evaporation of substance from the MP surface when the MP temperature is lower than boiling point and has the form $P_{\text {evpr }}^{-}=\Gamma_{a} \cdot\left(2 k_{B} T_{a}+p\right)$, where $\Gamma_{a}=n \sqrt{\frac{k_{B} T_{a}}{2 \pi m_{a}}} \exp \left(-\frac{p}{k_{B} T_{a}}\right)$ is the atom flow of evaporated MP substance, $n^{\prime}$ is the concentration of atoms in metal, $p$ is the energy of evaporation an atom, The results of numerical computations are shown in Fig. 5.

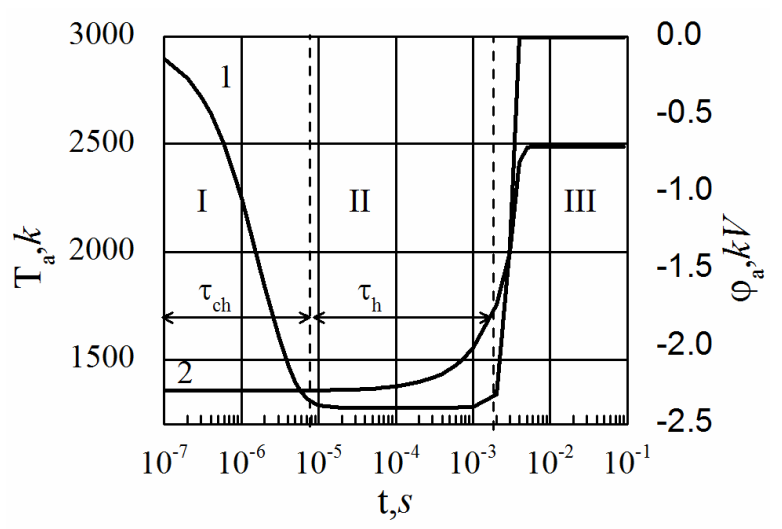

Fig. 5. Floating potential (1) and related temperature (2) of the copper MP as a function of the spent time in the plasma-beam system

$\left(n_{0}=10^{9} \mathrm{~cm}^{-3}, n_{b}=10^{9} \mathrm{~cm}^{-3}, E_{b}=5 \mathrm{keV}, a=10 \mu \mathrm{m}\right)$

The solution of the set of equations shows that the magnitude of the MP potential has being varied widely on time. It is supposed that when the MP had got into the plasma its charge was equal to 0 . Then in the region (I) "fast" charging $\left(\tau_{c h}\right)$ of the MP occurs. "Fast" means that MP has not warm up yet $\left(\tau_{c h}<\tau_{h}\right)$. Then in the region (II) magnitude of the MPs charge has not been changed that is $d \varphi_{a} / d t=0$, and it has maximum value. Then in the, region (III) the MP has warmed up to the temperature $T_{a}>T_{t h}$ when the magnitude of the MP charge potential decreases due to thermionic emission. Thus we conclude that the possibility of develop. 
Rayleigh instability is limited by the heating time $\tau_{h}$ to the temperatures $T_{a}>T_{t h}$ when the magnitude of the MP charge is decreasing.

$$
\tau_{\text {decay }} \approx \tau_{c h}+\tau_{0}<\tau_{h},
$$

where $\tau_{c h}$ is the charging time of the MP:

$$
\tau_{c h} \approx \frac{k E_{b}}{4 \pi a e^{2} n v_{e}} \ln \left(1+\frac{e \varphi_{a}}{k E_{b}}\right) .
$$

$\tau_{h}$ is the time when the MP has the maximum absolute magnitude of the MP charge:

$$
\tau_{h} \approx \frac{m_{a} c\left(T_{T h}-T_{0}\right)}{P^{\Sigma}}
$$

$\tau_{0}$ is the time of MP decay:

$$
\tau_{0}=\left(\sqrt{\beta-1} \sqrt{\frac{\alpha}{\rho}} a^{3 / 2}\right)^{-1},
$$

$\beta=E_{a} / E_{a}^{c r}>1$ - rate of overcharge of the MP, $\left|E_{a}^{c r}\right|=4 \sqrt{\frac{\pi \alpha}{a}}, \alpha$ is the surface tension, $a$ is the MP radius. The possibility of decay of various sized MP at different electron beam energies was also evaluated (Fig. 6).

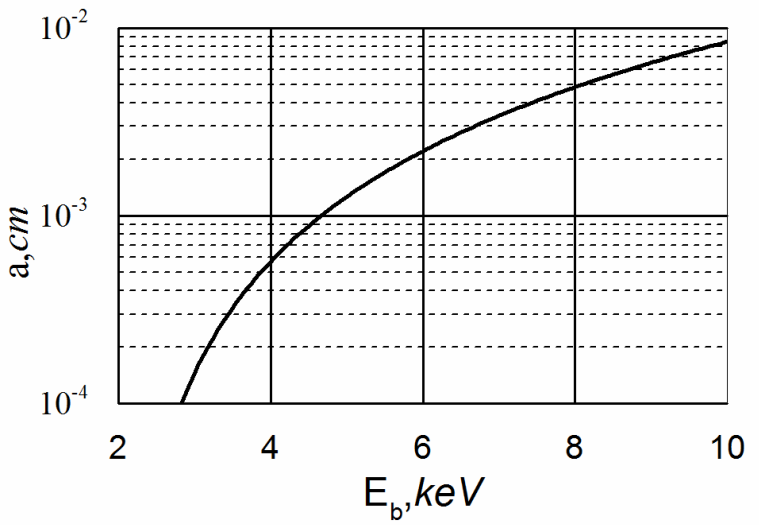

Fig. 6. The size of the MPs that can be destroyed as a result of their irradiation with an electron beam with a given energy

Thus in [7] it has been shown that MPs with sizes $a=1 . . .50 \mu \mathrm{m}$ that typical for technological plasma processes can be fragmented into smaller MPs due to irradiation with an electron beam of high energy $3 . . .8 \mathrm{keV}$. In [8], the possibility of evaporation of MPs in a hightemperature $\left(T_{e}=10 \ldots 100 \mathrm{eV}, T_{i}=1 \mathrm{eV}\right)$ technological, magnetized plasma has been studied.

If parameters of plasma and magnetic field such that:

$$
r_{g}>>\lambda_{d}>>a
$$

where $r_{g}$ is the Larmor radius of electron, $\lambda_{d}$ is the Debye length, $a$ is the MP radius. Then to describe fluxes of plasma onto the MP surface the OML theory can be used and set of equations have the form (1). Solution of the set of equations is shown in Fig. 7.

The analysis the solution revealed the cause of the unstable states of the MP, when in the regime of irradiation with an electron beam there was an abrupt change in the temperature of the MP. It was shown that at the bifurcation point, three different states of MPs are possible, which differ in temperature. This area is marked with a dashed line on the graph.

The reason for this effect is that at this moment the mechanism of thermionic emission is "switched on", which led to a sharp change in the potential of the MP, a change in the balance of currents on the MP and, as a consequence, of its temperature. A change in the MP temperature, in turn, can either enhance or weaken thermionic emission and cause the opposite effect, similar to positive feedback.
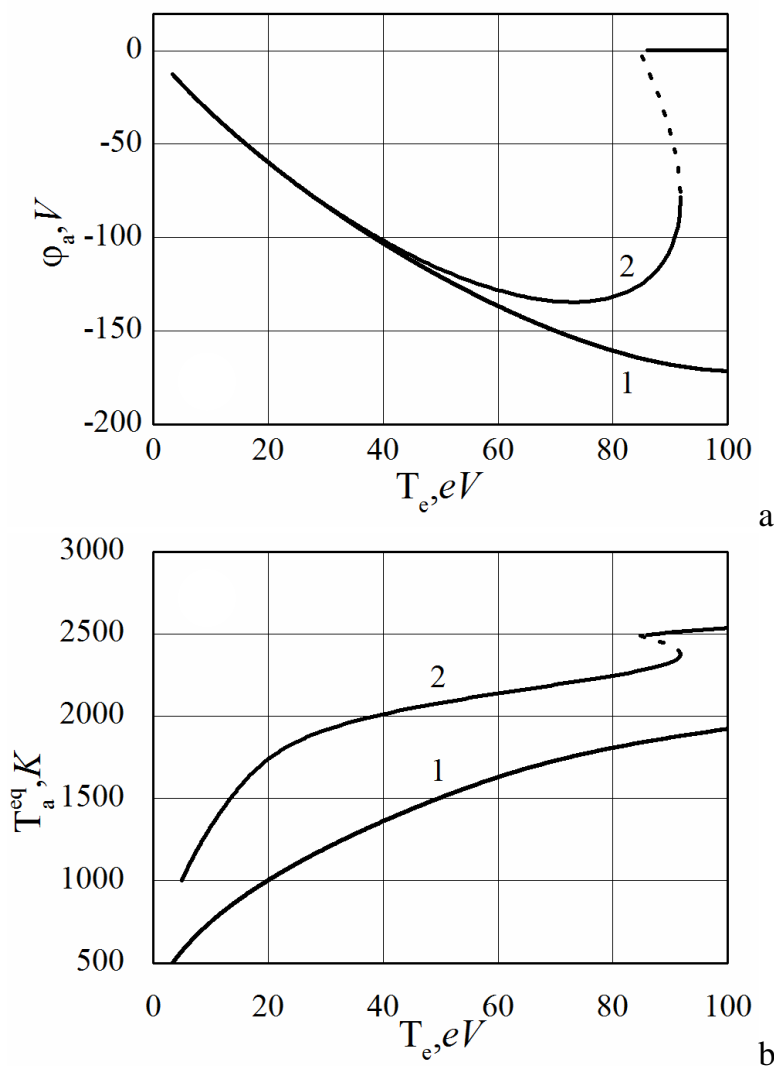

Fig. 7. Floating potential (1) and related (2)

Equilibrium temperature of the cooper MP as a function of electron temperature $\left(1-n_{0}=10^{10} \mathrm{~cm}^{-3}\right.$, $\left.2-n_{0}=10^{11} \mathrm{~cm}^{-3}\right)$

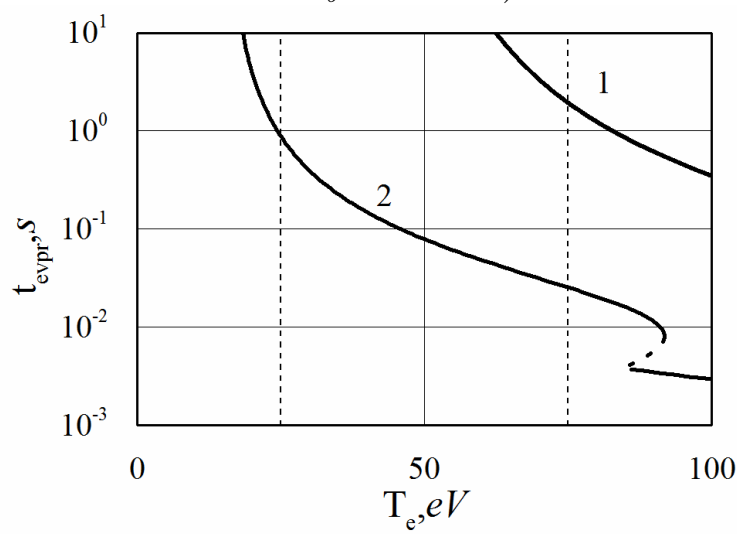

Fig. 8. The evaporation time of the cooper MP in the plasma $n_{0}=10^{11} \mathrm{~cm}^{-3}(1-a=10 \mu \mathrm{m}, 2-a=1 \mu \mathrm{m})$

In [8] has been also estimated the evaporation time (see Fig. 8) of the MP of radius a, that is given by the relation 


$$
t_{\text {evpr }}=\frac{4 \pi a^{3} \rho H}{3 P_{a}^{\Sigma}\left(T_{a}^{e q}, \varphi_{a}\right)},
$$

where $P_{e v p r}\left(T_{a}^{e q}, \varphi_{a}\right)=P_{e}^{+}+P_{i}^{+}-P_{\mathrm{s}}^{-}-P_{r}^{-}-P_{t h}^{-} \quad$ is the power that is spent on the evaporation of the MP substance. The mass loss of the MP during heating to the equilibrium temperature was neglected. From the Fig. 8 we can see that the evaporation time of the MP is decreased when the plasma temperature is increasing and at $100 \mathrm{eV}$ for MP of $1 \mu \mathrm{m}$ is $5 \cdot 10^{-3} \mathrm{~s}$ for the MP of $10 \mu \mathrm{m}$ is $5 \cdot 10^{-1} s$. Thus, if velocities of the MPs such that they pass through the region of heated plasma for time grater than obtained values it is possible fully evaporation of the MP.

\section{CONCLUSIONS}

1. Increasing of the plasma density as well as the electron beam energy lead to heating of the MP and to the appearance of the effect of thermionic emission, which leads to a decrease in the absolute value of its potential The effect of the thermionic emission from MP in plasma appear at the temperature of MP $T=2000 K$, which corresponds to the plasma density. $n_{0}=2 \cdot 10^{12} \mathrm{~cm}^{-3}$. In the presence of electron beam this effect occurs for beam energies $E_{b}=0.5 . .0 .7 \mathrm{keV}$. For such plasma and electron beam parameters, the MP potential decreases to zero.

2. Potential of the MP is largely determined by charge of ions. The ion energy has no significant effect on the MP potential in the usual energy range of arc discharges, whereas for two- and three-charged ions there is an effective heating of the MF due to the recombination of ions on its surface.

3. There is a certain range of values of the electron plasma temperature, where there are three values of the stationary temperature of the MP, and the average value of them is unstable with fluctuations in the MP temperature. The stationary temperature of the MP and its potential in this region are determined by the initial temperature of the MP.

4. The characteristics of the MP (size and velocity), which can evaporate when passing through a heated plasma, have been determined.

5. There is a time interval in which the conditions for Rayleigh instability of a liquid MP are satisfied. This interval is determined by the heating time interval from the initial temperature to temperature of $1700 \ldots 2000 \mathrm{~K}$, at which the discharge of MP occurs due to the "switch on" of thermionic emission. For the MPs, size of which is about $a \sim 1 \mu \mathrm{m}$ the decay time is approximately equal to the MP time of charge, for more large MPs $a>10 \mu m$ decay time is approximately equal to the time of the development Rayleigh instability. For MP with $a \sim 10 \mu m$ the conditions for Rayleigh instability are optimal.

\section{REFERENCES}

1. V.E. Fortov, A.G. Khrapak, et al. Dusty plasma // UFN. 2004, v. 174, № 5, p. 495-544.

2. P.K. Shucla, A. Mamun. Introduction to Dusty Plasma Physics. Bristol: "IOP Publishing”, 2002.

3. R.L. Boxman, S. Goldsmith. Macroparticle contamination in cathodic arc coatings: generation, transport and control // Surf. Coat. Thechnol. 1992, v. 52, p. 39-50.

4. I.I. Aksenov, A.A. Andreev, et al. Vacuum Arc: plasma sources, coating deposition, surface modification. Kiev: "Naukova Dumka", 2012.

5. A.A. Bizyukov, A.D. Chibisov, E.V. Romashchenko. Effect of the parameters of a gas-discharge plasma on the equilibrium temperature and floating potential of macroparticle // Problems of Aomic Science and Technology. Series «Plasma Physics» (18). 2012, № 6, p. 175-177.

6. A.A. Bizyukov, A.D. Chibisov, et. al. Charging processes and phase states of macroparticles in lowpressure arc discharge// Problems of Atomic Science and Technology. Series «Plasma Electronics and New Methods of Acceleration» (8). 2013, № 4, p. 176-178.

7. A.A. Bizyukov, A.D. Chibisov, E.V. Romashchenko, Yu.E. Kolyada. Decay of liquid metallic macroparticles in plasma-beam systems due to rayleigh instability // Problems of Atomic Science and Technology. Series «Plasma Physics» (23). 2017, № 1, p. 163-166.

8. A.A. Bizyukov, A.D. Chibisov, et al. Vaporization of metallic macroparticles in the high temperature technology plasma // Problems of Atomic Science and Technology. Series «Plasma Physics» (22). 2016, № 6, p. $268-271$.

Article received 05.01.2021

\section{ОСОБЕННОСТИ ВЗАИМОДЕЙСТВИЯ МАКРОЧАСТИЦ С ПЛАЗМЕННО-ПУЧКОВЫМИ СИСТЕМАМИ}

\section{А.А. Бизюков, А.Д. Чибисов, Е.В. Ромащенко, В.В. Масич}

Обобщены результаты последних работ авторов по моделированию процессов нагрева и испарения макрочастиц в плазменно-пучковой системе. Особое внимание уделяется влиянию параметров плазмы, а также электронного пучка, вводимого в плазму, на эти процессы. Был проведен подробный анализ эффектов, которые ускоряют или замедляют скорость нагрева и испарения макрочастиц.

\section{ОСОБЛИВОСТІ ВЗАСМОДІЇ МАКРОЧАСТОК 3 ПЛАЗМОВО-ПУЧКОВИМИ СИСТЕМАМИ}

\section{О.А. Бізюков, О.Д. Чібісов, О.В. Ромащенко, В.В. Масич}

Узагальнено результати останніх робіт авторів з моделювання процесів нагрівання і випаровування макрочасток у плазмово-пучковій системі. Особлива увага приділяється впливу параметрів плазми, а також електронного пучка, що вводиться в плазму, на ці процеси. Було проведено детальний аналіз ефектів, які прискорюють або уповільнюють швидкість нагріву і випаровування макрочасток. 